\title{
Odorant Concentration Differentiator for Intermittent Olfactory Signals
}

\author{
Terufumi Fujiwara, ${ }^{1}$ Tomoki Kazawa, ${ }^{2}$ Takeshi Sakurai, ${ }^{2}$ Ryota Fukushima, ${ }^{2,3}$ Keiro Uchino, ${ }^{4}$ Tomoko Yamagata, ${ }^{2,3}$ \\ Shigehiro Namiki, ${ }^{2}$ Stephan Shuichi Haupt, ${ }^{2,5}$ and Ryohei Kanzaki ${ }^{1,2}$ \\ ${ }^{1}$ Graduate School of Information Science and Technology, University of Tokyo, Tokyo, 113-8656, Japan, ${ }^{2}$ Research Center for Advanced Science and \\ Technology, University of Tokyo, Tokyo, 153-8904, Japan, ${ }^{3}$ Graduate School of Life and Environmental Sciences, University of Tsukuba, Ibaraki, 305-8572, \\ Japan, ${ }^{4}$ Transgenic Silkworm Research Unit, National Institute of Agrobiological Sciences, Ibaraki, 305-8634, Japan, and ${ }^{5}$ Department of Biological \\ Cybernetics, Faculty of Biology, Bielefeld University, 33615 Bielefeld, Germany
}

Animals need to discriminate differences in spatiotemporally distributed sensory signals in terms of quality as well as quantity for generating adaptive behavior. Olfactory signals characterized by odor identity and concentration are intermittently distributed in the environment. From these intervals of stimulation, animals process odorant concentration to localize partners or food sources. Although concentration-response characteristics in olfactory neurons have traditionally been investigated using single stimulus pulses, their behavior under intermittent stimulus regimens remains largely elusive. Using the silkmoth (Bombyx mori) pheromone processing system, a simple and behaviorally well-defined model for olfaction, we investigated the neuronal representation of odorant concentration upon intermittent stimulation in the naturally occurring range. To the first stimulus in a series, the responses of antennal lobe (AL) projection neurons (PNs) showed a concentration dependence as previously shown in many olfactory systems. However, PN response amplitudes dynamically changed upon exposure to intermittent stimuli of the same odorant concentration and settled to a constant, largely concentration-independent level. As a result, PN responses emphasized odorant concentration changes rather than encoding absolute concentration in pulse trains of stimuli. Olfactory receptor neurons did not contribute to this response transformation which was due to long-lasting inhibition affecting PNs in the AL. Simulations confirmed that inhibition also provides advantages when stimuli have naturalistic properties. The primary olfactory center thus functions as an odorant concentration differentiator to efficiently detect concentration changes, thereby improving odorant source orientation over a wide concentration range.

Key words: adaptation; insect; olfaction; sensory intensity

\section{Introduction}

Animals use concentration information to approach attractive odorant sources (Baker and Roelofs, 1981; Gomez-Marin et al.,

Received June 6, 2014; revised Sept. 26, 2014; accepted 0ct. 21, 2014.

Author contributions: T.F., T.K., and S.S.H. designed research; T.F., T.S., R.F., K.U., T.Y., and S.S.H. performed research; T.F., T.K., S.N., and S.S.H. analyzed data; T.F., T.K., T.S., S.S.H., and R.K. wrote the paper.

This work was supported by Research and Development of the Next-Generation Integrated Simulation of Living Matter (part of the Development and Use of the Next-Generation Supercomputer Project), a Grant-in-Aid for Scientific Research (B) (21370029 and 24370031), a Japan Society for the Promotion of Science postdoctoral scholarship (P07144), and a Grant-in-Aid for Japan Society for the Promotion of Science fellows (22-6410) from the Ministry of Education, Culture, Sports, Science and Technology. We thank Professor Shigeru Matsuyama (University of Tsukuba) for providing purified bombykol; Dr. Junichi Nakai (Saitama University Brain Science Institute) for plasmid DNA containing GCaMP2; Dr. Toshiki Tamura (National Institute of Agrobiological Sciences) and Dr. Hideki Sezutsu (National Institute of Agrobiological Sciences) for generation of UAS-GCaMP2 moths; and Dr. Eugenia Chiappe for helpful comments on the manuscript.

The authors declare no competing financial interests.

Correspondence should be addressed to either of the following: Dr. Ryohei Kanzaki or Dr. Stephan Shuichi Haupt, Research Center for Advanced Science and Technology, University of Tokyo, 4-6-1 Komaba, Meguro-ku, Tokyo, 153-8904, Japan. E-mail: kanzaki@rcast.u-toky0.ac.jp or stephan.haupt@uni-bielefeld.de.

T. Fujiwara's present address: Champalimaud Neuroscience Programme, Champalimaud Centre for the Unknown, Av. Brasília, Doca de Pedrouços, 1400-038, Lisboa, Portugal.

T. Yamagata's present address: Frontal Lobe Function Project, Tokyo Metropolitan Institute of Medical Science, 2-1-6 Kamikitazawa, Setagaya-ku, Tokyo 156-8506, Japan.

S. Namiki's present address: Janelia Farm Research Campus, Howard Hughes Medical Institute, 19700 Helix Drive, Ashburn, VA 20147.
2011; Khan et al., 2012; Takasaki et al., 2012). To reveal how olfactory neurons encode intensity information, concentrationresponse characteristics of a wide variety of neuron-odor combinations have been studied in different animals (Boeckh and Boeckh, 1979; Kanzaki and Shibuya, 1986; Friedrich and Korsching, 1997; Wang et al., 2003; Hallem and Carlson, 2006; Meeks et al., 2010; Fujiwara et al., 2014). Neuronal responses generally show a concentration-dependent increase for well-separated single stimulus odorant pulses. In natural environments, however, olfactory signals are discontinuously distributed in space and time, resulting in short intervals between stimuli (Justus et al., 2002). The dynamics of concentration-response characteristics of neurons under these relevant conditions are largely unknown. Male moth pheromone processing systems have been investigated as convenient models of odorant source orientation due to the well-defined behaviors they generate and the quantitative simplicity of the neuronal circuits (Hildebrand, 1996). In male silkmoths, (E,Z)-10,12-hexadecadienol (bombykol), the major female sex pheromone component, is sufficient to trigger male orientation behavior (Butenandt et al., 1959). Olfactory receptor 
neurons (ORNs) expressing BmOR1, the bombykol receptor, deliver bombykol information to the toroid, a large glomerulus of the macroglomerular complex (MGC) in the antennal lobe (AL), the primary olfactory center (Sakurai et al., 2004, 2011). In the toroid, information is processed by projection neurons (PNs) (Kanzaki and Shibuya, 1983; Kanzaki et al., 2003) and inhibitory local interneurons (LNs) (Iwano and Kanzaki, 2005; Seki and Kanzaki, 2008).

To understand how it is possible that moths discriminate concentrations of stimuli that are received sequentially, we investigated the dynamics of concentration-response characteristics of ORNs and AL PNs innervating the toroid using intermittent bombykol stimuli. The responses of ORNs were measured in the axonal arborizations directly presynaptic to the PNs taking advantage of the accessibility of transgenesis in silkmoths by expressing GCaMP, a genetically encoded calcium indicator, exclusively in the bombykol responsive ORNs. The difference in response characteristics between first- and second-order neurons revealed a novel transformation of odorant concentration coding downstream of the ORN-PN synapses in the AL, which was due to inhibitory circuitry in the AL. These inhibitory mechanisms in the AL are involved in transforming the encoding of absolute odorant concentration in ORNs into an encoding of relative concentrations in PNs to allow efficient detection of concentration differences upon exposure to intermittent stimuli. To test the significance of inhibition for concentration discrimination in the presence of naturalistic stimuli composed of fluctuating odorant concentrations and interstimulus intervals, we implemented a model of AL reflecting the inhibitory properties we identified in physiological experiments. Using dynamic stimulus inputs inferred from electroantennogram (EAG) responses to a female releasing pheromone, we show that inhibition within the AL improves odorant concentration discrimination in $\mathrm{PN}$ responses under natural stimulus conditions.

\section{Materials and Methods}

Animals. Male and female silkmoth larvae (Bombyx mori L.) were reared on an artificial diet (Silk Mate 1-3S; Nosan Bio Department) at $26^{\circ} \mathrm{C}$ and $60 \%$ relative humidity under a 16:8 h (light/dark) light cycle or bought at pupal stage (Nikko Marketing). The imagines were used 1-6 d after eclosion.

Vector construction and generation of a transgenic moth line. Plasmid DNA containing GCaMP2 (pN1-GCaMP2) (Tallini et al., 2006) was kindly provided by Dr. J. Nakai of Saitama University. The HindIIIEcoRI fragment of pUAST (Brand and Perrimon, 1993) containing the upstream activation sequence (UAS) and TATA element of the Drosophila melanogaster was inserted into corresponding sites of pEGFP-N1 (Clontech) to create pUAS-EGFP-N1. The BglII-NotI fragment of pN1GCaMP2 that contains GCaMP2 and SV40 polyadenylation signal sequence was inserted into BamHI-NotI sites of pUAS-EGFP-N1. The resulting vector was named pUAS-GCaMP2-N1, obtained by replacing EGFP in pUAS-EGFP-N1 by GCaMP2. pUAS-GCaMP2-N1 was digested with BgIII and AflII, and the AflII restriction end was ligated with adapter DNA (TTAATAGATCTA) that contained a BglII site. Then, the ligated DNA was digested with BglII and subcloned into the BgllII site of pBacMCS2 containing additional PmeI and FseI restriction sites immediately upstream of the ClaI site of pBacMCS (Uchino et al., 2006) to create pBacUAS-GCaMP2. The DNA fragment for a screening marker that consists of fibroin light-chain (FibL) promoter, DsRed monomer, and 3'UTR of $f i b L$ gene (FibL-DsRed) was amplified using PrimeStar DNA polymerase (Takara), pBacUAS-PxOR1 (Sakurai et al., 2011) as template and the primers 5' $5^{\prime}$ TGCTAGCCACTAAAGGGAACAAAAGCTGG-3' and 5'TGCTAGCCACTATAGGGCGAATTGGGTACC-3'. The DNA fragment was inserted into the BamHI site of pBacUAS-GCaMP2 after the site was blunted with T4 DNA polymerase (Takara). The resultant vector was named pBacUAS-GCaMP2-FibLDsRed (see Fig. 4A).
Transgenic silkmoths were generated using the piggyBac-mediated germline transformation method, as described previously (Tamura et al., 2000, 2007). To direct the expression of GCaMP2 in bombykol receptor neurons, UAS-GCaMP2 moths were crossed with BmOR1-GAL4 moths that express GAL4 in bombykol receptor neurons (Sakurai et al., 2011). Progeny carrying both UAS-GCaMP2 and BmOR1-GAL4 transgenes was used for calcium imaging experiments.

The brains of the BmOR1-GAL4/UAS-GCaMP2 male moths were fixated overnight at $4^{\circ} \mathrm{C}$ in $4 \%$ depolymerized PFA in PBS, rinsed in several changes of PBS containing $0.2 \%$ Triton X-100 (PBTX) and preincubated with $5 \%$ normal donkey serum and 5\% normal goat serum in PBTX (PBTX-NDS-NGS) for $3 \mathrm{~h}$ at room temperature. Then, they were incubated with mouse anti-synaptotagmin monoclonal antibody (1:100) in PBTX-NDS-NGS at $4^{\circ} \mathrm{C}$ for $3 \mathrm{~d}$. The mouse anti-synaptotagmin monoclonal antibody (3H2 2D7) developed by K. Zinn was obtained from the Developmental Studies Hybridoma Bank developed under the auspices of the National Institute of Child Health and Human Development and maintained by the University of Iowa, Department of Biology (Iowa City, Iowa). Next, the brains were rinsed in PBTX and incubated with Cy3conjugated anti-mouse IgG antibodies (1:200; Jackson ImmunoResearch Laboratories) in PBTX-NDS-NGS at $4^{\circ} \mathrm{C}$ overnight. The labeled brains were viewed under a confocal laser scanning microscope (LSM510; Carl Zeiss). GCaMP2 was excited with an argon laser at $488 \mathrm{~nm}$, and fluorescence was recorded with a 505-550 $\mathrm{nm}$ bandpass filter. Cy3 was excited with a HeNe laser at $543 \mathrm{~nm}$, and fluorescence was recorded with a 560 $\mathrm{nm}$ long-pass filter. Serial optical sections were acquired in $0.8 \mu \mathrm{m}$ intervals and at a size of $512 \times 512$ pixels. Confocal images were adjusted for contrast and brightness using Adobe Photoshop CS (Adobe Systems).

Animal preparation. The abdomen, legs, and dorsal side of the thorax were removed, and the moths were mounted in a plastic holder. The brain was exposed and superfused with saline solution (140 mM NaCl, 5 mм KCl, $7 \mathrm{~mm} \mathrm{CaCl}_{2}, 1 \mathrm{~mm} \mathrm{MgCl}_{2}, 4 \mathrm{~mm} \mathrm{NaHCO}_{3}, 5 \mathrm{~mm}$ trehalose, $5 \mathrm{~mm}$ $\mathrm{N}$-tris [hydroxymethyl] methyl-2-aminoethanesulfonic acid, and 100 mm sucrose, $\mathrm{pH}$ 7.0). The intracranial muscles, parts of the compound eyes, and the tracheae around the AL were removed, and the AL was surgically desheathed.

EAG recording. Glass micropipettes with silver chloride wire were filled with Ringer's solution and inserted into the distal and proximal ends of a male antenna mounted in a portable chamber. To protect them from drying, the cut ends of the antenna were covered by gel (SPECTRA 360; Parker Laboratories). At first, we positioned the portable chamber in front of our odor delivery system, which was used in other physiological experiments, and measured EAG responses to synthetic bombykol. The EAG responses were amplified (MEZ-8300; Nihon Kohden), low-pass filtered at $5 \mathrm{kHz}$, and digitized at $10 \mathrm{kHz}$ (USB-6009; National Instruments). Then, we moved the portable chamber to a wind tunnel and positioned it $10 \mathrm{~cm}$ downwind from a female to measure EAG responses to female-emitted pheromones. The wind tunnel was made of Styrofoam boards (Dow Chemical, W $840 \mathrm{~mm} \times \mathrm{H} 300 \mathrm{~mm} \times \mathrm{L} 1800 \mathrm{~mm}$ ) and connected to an exhaust duct to produce a constant air flow of $0.5 \mathrm{~m} / \mathrm{s}$ average speed. Voltage was amplified and low-pass filtered at $5 \mathrm{kHz}$ by a custom made amplifier (S.S.H.) and digitized at $10 \mathrm{kHz}$ (USB-6215, National Instruments). After the recording in the wind tunnel, we measured the responses to synthetic bombykol again and discarded samples in which the response amplitude to the strongest bombykol stimulus was decreased by $>40 \%$ compared with the first measurement.

Physiology. Imaging was performed with a CCD camera (iXon ${ }^{\mathrm{EM}+}$ EMCCD DU-897E; Andor Technology) using a 20× (XLUMPFLN 20XW, NA 1.0) water-immersion lens on a fluorescence microscope (BX51WI; Olympus) with U-MWIBA3 filter set (Olympus). The exposure time was $100 \mathrm{~ms}$. The interval between trials was at least $1 \mathrm{~min}$. For loosepatch recording, the preparation was treated with enzymes (Kloppenburg et al., 1999) (collagenase, 038-10531; Wako, $0.5 \mathrm{mg} / \mathrm{ml}$, dispase, D4693; Sigma, $2 \mathrm{mg} / \mathrm{ml}$ ) at room temperature for 5-10 min. The recording of PNs was performed using micropipettes $(3-5 \mathrm{M} \Omega$ ) filled with AlexaFluor-568 (A-10441; Invitrogen, $0.1 \mathrm{~mm}$ in saline) to visualize the tip. Voltage was amplified, filtered, and digitized in the same way as EAG responses.

Stimulation. Synthetic bombykol, the principal pheromone component of B. mori, was dissolved in n-hexane. A glass cartridge ( $5 \mathrm{~mm}$ inner 
diameter) was prepared for stimulation by inserting a piece of filter paper $(1 \times 2 \mathrm{~cm})$ containing $5 \mu \mathrm{l}$ of solution of the dissolved odorant or n-hexane as control. The odorant was delivered by switching a $3 / 2$ solenoid valve (YDV-3-1/8; Takasago Electric) with constant air flow (1 $1 / \mathrm{min}$ ) from control cartridge to pheromone cartridge.

Pharmacology. Picrotoxin (PTX, 168-17961; Wako) was prepared as a $250 \mathrm{~mm}$ stock in DMSO and used at a final concentration of $50 \mu \mathrm{M}$ in silkmoth ringer. Neuronal responses were measured after $10 \mathrm{~min}$ bath application of the drug. Recovery was not tested because of the considerable amount of time required to wash out drugs (Waldrop et al., 1987), in particular hydrophobic compounds, such as PTX, applied at elevated concentrations to block an appreciable fraction of inhibition.

Data analysis. Data were processed using MATLAB (MathWorks) and Excel (Microsoft). For imaging, per-pixel baseline fluorescence was determined as an average over 10 frames $(1 \mathrm{~s})$ before stimulation, and relative fluorescence changes $(\Delta \mathrm{F} / \mathrm{F})$ with respect to the baseline fluorescence were calculated. Data were spatially averaged in ROIs defined as the pixels 2 SD brighter than the average of all pixels. The exponential decay due to bleaching was removed by subtracting time courses recorded using the control stimulus. ORNs did not respond to the control stimulus. A Gaussian filter was applied to the color-coded images with a five pixel kernel $(\sigma=1)$. Values were normalized on the maximum response within each preparation. Loose-patch recording data of PNs were highpass filtered at $100 \mathrm{~Hz}$ and smoothed (5 point moving average). The responses to bombykol were always phasic, and spontaneous spikes were exceptional; therefore, we could simply calculate the peak instantaneous spike frequency and spike counts for each stimulus. Spike response duration was defined as the duration between the time of first and last spikes to an odorant stimulus. The data from EAG recordings were bandpass filtered $(0.03-10 \mathrm{~Hz})$ and smoothed (moving average for $0.1 \mathrm{~s})$. To extract single-response events putatively induced by single odorant filaments from the EAG responses measured in the wind tunnel, the data were additionally low-pass filtered $(2 \mathrm{~Hz})$ and local minima were defined as peaks of each response event by thresholding based on the slope of the rising and falling phases. A time interval of $200 \mathrm{~ms}$ before a peak was defined as the rising phase; the first $500 \mathrm{~ms}$ after a peak was defined as the falling phase. The average value of data points preceding rising phases around a peak by $>200 \mathrm{~ms}$ and $>500 \mathrm{~ms}$ after falling phases around a peak was defined as baseline. The amplitude of each response event was calculated by subtracting the baseline voltage from the peak voltage. The rising slope of each response event was calculated by fitting the points in the rising phase to a linear function. For analyzing the EAG responses to synthetic bombykol pulses, the mean voltage over $1 \mathrm{~s}$ before the stimulus onset was defined as baseline. The amplitude and the rising slope were calculated as described above. For the responses to synthetic bombykol, we averaged the values of the trials before and after the recording in the wind tunnel. All values were normalized on the highest response to synthetic bombykol within each preparation. Normalized responses to synthetic bombykol and single-response events to pheromone released by a female were compared.

For comparing ORN- and PN-evoked response levels between stimulus concentrations in the settled state occurring at the end of stimulus pulse trains, we defined "average settled response level" as the average of responses to the last 10 stimuli in the pulse train, calculated for each individual experiment. For comparing concentration-response relations, we defined "concentration dependence" as the slope of individual concentration-response curves. These curves consist of 3 points (odorant concentrations) for intermittent stimuli.

Simulation. In the model, a PN has an excitatory conductance $\left(g_{e}\right)$ activated by odorant-induced ORN activity and an inhibitory conductance $\left(g_{i}\right)$ activated by LN input. The summation of the two conductances is transformed into PN peak firing rate $\left(R_{P N}\right)$ (see Fig. $7 \mathrm{~A}$ ) by the transfer function $F$ as follows:

$$
R_{P N}=F\left(g_{e}+g_{i}\right)
$$

Excitatory and inhibitory conductances were described as convolutions of stimulus-induced response amplitudes of ORNs $\left(R_{O R N}\right)$ or LNs $\left(R_{L N}\right)$ with exponential functions:

$$
\begin{gathered}
g_{e}=\left.R_{\text {ORN }} \otimes \exp \left(-\frac{t}{\tau_{e}}\right)\right|_{t>0} \\
g_{i}=-\left.R_{L N} \otimes \exp \left(-\frac{t-t_{\text {delay }}}{\tau_{i}}\right)\right|_{t>t_{\text {delay }}}
\end{gathered}
$$

where $t$ is time (in $10 \mathrm{~ms}$ bins), $\tau_{e}$ is the time constant of $g_{e}(200 \mathrm{~ms}), t_{\text {delay }}$ and $\tau_{i}$ are the onset delay $(100 \mathrm{~ms})$ and the time constant of $g_{i}(1200 \mathrm{~ms}$, except for Fig. $7 I$, where the time constant was varied in the range of $0.1-2 \mathrm{~s})$

The transfer function $F$ was described as a Hill equation (see Fig. $7 B$ ) as follows:

$$
F(x)=\left\{\begin{array}{c}
\frac{R_{P N} \max }{1+\left(\frac{E C_{50 P N}}{\max (x)}\right)^{n_{P N}}}(x>0) \\
0(x \leq 0)
\end{array}\right.
$$

where $\max (x)$ is the local maximum of $x$ between the onset of a stimulus and the onset of the following stimulus. PN maximum firing rate $\left(R_{P N} \max\right.$ ) was set to $270 \mathrm{~Hz}$ based on published data (Fujiwara et al., 2014). The Hill coefficient $\left(n_{P N}=1.27\right)$ and the half-maximal effective concentration $\left(E C_{50 P N}=0.178\right)$ were determined by fitting (using the MATLAB nlinfit function, $R^{2}=0.9993$ ) the dataset of the average ORN response amplitudes to the first stimulus shown in Figure 4 (for $x$ ) and average PN peak firing rates to the first stimulus shown in Figure 2 (for $F(x)$ ) to the function $F$ based on the assumption that $\mathrm{PN}$ peak firing rates to the first stimulus are exclusively resulting from activation by the excitatory conductance component.

$R_{L N}$ was also defined as a Hill equation (see Fig. $7 D$ ) as follows:

$$
R_{L N}=\frac{R_{L N} \max }{1+\left(\frac{E C_{50 L N}}{R_{O R N}}\right)^{n_{L N}}}
$$

The values of LN maximum response amplitude $\left(R_{L N} \max =2.3\right)$, Hill coefficient $\left(n_{L N}=1.7\right)$, and half-maximal effective concentration $\left(E C_{50 L N}=0.9\right)$ were determined by minimizing that the difference of simulated PN peak firing rates (using ORN response amplitudes from Fig. 4 as input) and actual PN peak firing rates (see Fig. 2) to intermittent stimuli summing over all stimulus pluses and concentrations (see Fig. $7 C, D)$.

Using these parameters, we simulated $\mathrm{PN}$ responses to naturalistic odorant inputs that contain fluctuations in stimulus concentration and interval (see Fig. $7 H$ ). EAG responses to females emitting pheromones from 8 datasets were used as inputs (see Fig. 1). Response amplitudes of single EAG components were linearly transformed into $R_{O R N}$ by scaling such that $R_{O R N}$ to an odorant stimulus of a concentration produced by $1000 \mathrm{ng}$ bombykol corresponded to the mean response amplitude of single EAG components. An odorant concentration discrimination score was defined as the average PN peak firing rate increase (decrease) for odorant concentration increase (decrease) events in the time sequence of EAG responses. These scores were normalized by $R_{P N} \max$. In the model without inhibition, $\mathrm{PN}$ peak firing rates were calculated exclusively based on the excitatory conductance component.

Unless otherwise noted, a one-way repeated-measures ANOVA followed by the Tukey-Kramer test was used to compare among multiple groups, and the Wilcoxon signed-rank test was used to compare between two groups. Data are shown as mean \pm SEM, unless indicated otherwise.

\section{Results}

\section{Estimating the concentration range of}

\section{female-emitted bombykol}

To use behaviorally relevant stimuli for characterizing neurons, we investigated the concentration range of bombykol that male silkmoths encounter under natural circumstances. Using EAGs from male antennae, we compared synthetic bombykol concentrations delivered by our stimulator system to the amount of 
female-emitted pheromone. The EAG amplitude monotonically increased with increasing concentration of synthetic bombykol (Fig. 1A). Using the same antennae, we also recorded EAG responses to pheromone emitted by females (Fig. $1 B)$. We detected individual response events from the EAG, each of which is supposed to be induced by a single odorant filament. The maximum amplitudes for single events corresponded to responses elicited by 1000-5000 ng synthetic bombykol in our stimulator system (Fig. 1C). The mean amplitudes of singleresponse events were comparable with the responses to stimulation with $1000 \mathrm{ng}$ bombykol (Fig. 1C). Results were similar when using EAG response rising slopes for the analysis (Fig. 1D). Although femaleemitted pheromones contain a second component, bombykal, it can be omitted in EAG responses because of its small fraction (Kaissling and Kasang, 1978). According to these results, we defined 1000-2000 ng as "high" stimulus concentration in the following experiments. For convenience, we defined $10 \mathrm{ng}$ as the "low" concentration and $100 \mathrm{ng}$ as a "medium" concentration. The frequency of the EAG individual events was $0.79 \pm 0.05 \mathrm{~Hz}(n=8$, mean \pm SEM), and we therefore used a comparable stimulus interval $(1200 \mathrm{~ms}$, corresponding to $0.83 \mathrm{~Hz}$ ) in the following experiments.

\section{Dynamic representation of odorant concentration in AL PNs}

We investigated how bombykol concentration is represented upon exposure to intermittent stimuli at the output of the AL (Fig. 2A). We recorded spike responses of PNs innervating the toroid (henceforth just PNs) to bombykol pulse trains of three concentrations by loose-patch voltage recording (Fig. 2A). PNs showed phasic spike trains to each stimulus pulse (Fig. $2 B$ ). In response to the first stimulus, the peak instantaneous frequency (peak ISF) increased as the concentration increased (Fig. 2C,D; $F_{(2,5)}=43.24$, Tukey-Kramer test: $p<0.05$ among all concentrations, $n=6)$. For spike counts, there were no significant differences among the concentrations (Fig. $2 C, E ; F_{(2,5)}=0.59$, $p=0.57, n=6$ ). Responses showed dynamic transitions for subsequent stimuli (Fig. 2C-E). At low concentration, the peak ISF and the spike counts decreased upon the second stimulus and settled to a relatively constant level for subsequent stimuli. At medium and high concentrations, once PNs received a bombykol stimulus, the responses to the subsequent stimuli were largely suppressed. The responses gradually recovered over the following stimulus pulses and settled to a stable level. Once settled, responses were concentration-independent for medium and high concentrations (Fig. $2 C-E ; F_{(2,5)}=13.39$, Tukey-Kramer test: $p>0.05$ for average settled response levels to 100 and $1000 \mathrm{ng}$ for both peak ISF and spike counts, $n=6$ ). This can be likened to the action of a differentiator. Early response suppression was not due to concentration decrease related to cartridge stimulation because EAG response amplitudes did not significantly change over stimulus pulses with our odorant delivery system (Fig. 2F; peak
B

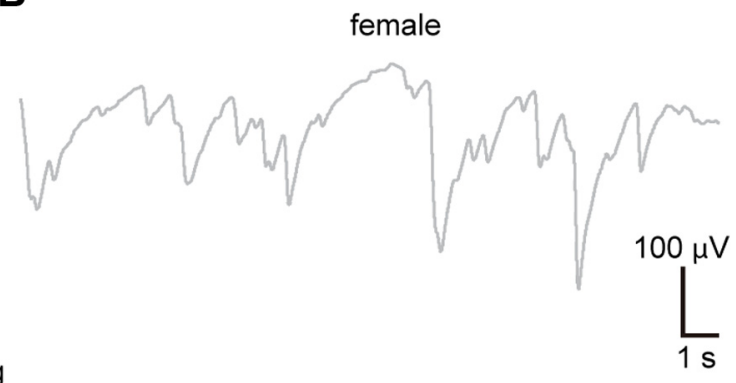

D

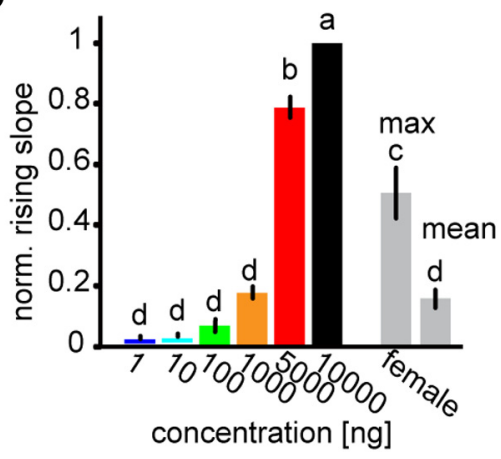

concentration [ng]

a

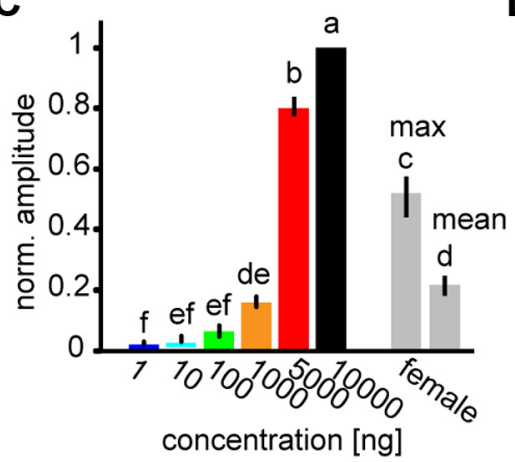

Figure 1. EAG responses to synthetic bombykol and females emitting pheromones. $A$, EAG responses to synthetic bombykol pheromones emitted by females. For pheromones emitted by females, maximum and mean responses of the single EAG re shown (one-way repeated-measures ANOVA): for amplitudes, $F_{(7,7)}=170.28, p<0.001$; for rising slopes, $F_{(7,7)}$ 119.00, $p<0.001$. $p<0.05$, significant differences indicated by different letters associated with the data groups (TukeyKramer test). Data are means; error bars indicate SEM; $n=8$.

EAG responses were $82.1 \pm 14.7 \mu \mathrm{V}$ for first stimulus pulse and $90.2 \pm 16.1 \mu \mathrm{V}$ for 14 th stimulus pulse, $p=0.20, n=9$; antenna was covered by a glass deflector between the two measurements).

For single odorant stimulus pulses, ORNs show concentrationindependent response dynamics while representing concentration information in their response amplitudes (Martelli et al., 2013), suggesting the possibility of different concentration representations in response dynamics and response amplitudes. We also investigated PN response dynamics in the settled state (Fig. 2G). Response duration was shorter at higher compared with lower stimulus concentrations $(64.2 \pm 11.0,54.9 \pm 7.0$, and $50.1 \pm 7.0$ $\mathrm{ms}$ at 10,100 , and $1000 \mathrm{ng}$, respectively; $F_{(2,5)}=0.59$, TukeyKramer test: $p<0.05$ between 10 and $1000 \mathrm{ng}, n=6)$ and response latency decreased in a concentration-dependent manner $(360.9 \pm 3.7,339.2 \pm 4.1$, and $322.4 \pm 4.4 \mathrm{~ms}$ at 10,100 , and $1000 \mathrm{ng}$, respectively; $F_{(2,5)}=57.80$, Tukey-Kramer test: $p<0.05$ among all concentrations, $n=6$ ). Therefore, absolute concentration information could be still relayed to downstream circuitry via response latency.

\section{Functional role of the transformation of odorant concentration representation}

After the transformation of concentration representation under intermittent stimulation, $\mathrm{PN}$ responses do not seem to represent absolute bombykol concentration as response levels in the settled state are largely independent of concentration. Then, what is represented after the transformation? One possibility is that the parameter odorant concentration is differentiated in $\mathrm{PN}$ responses, maintained at a concentration-independent level, whereas pulse stimulus concentration remains constant among pulses but changing dramatically upon concentration changes. This could 

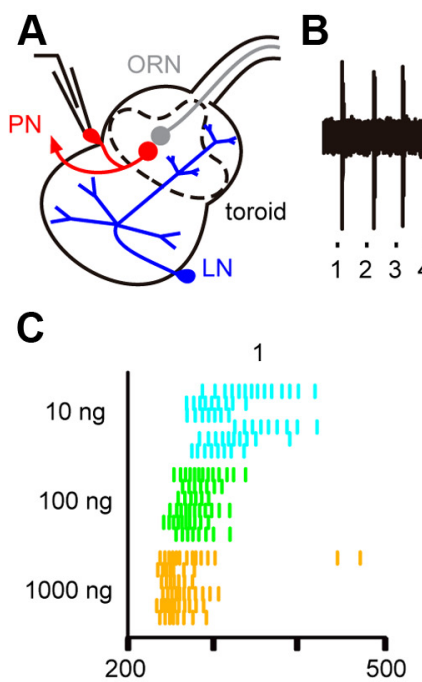

C
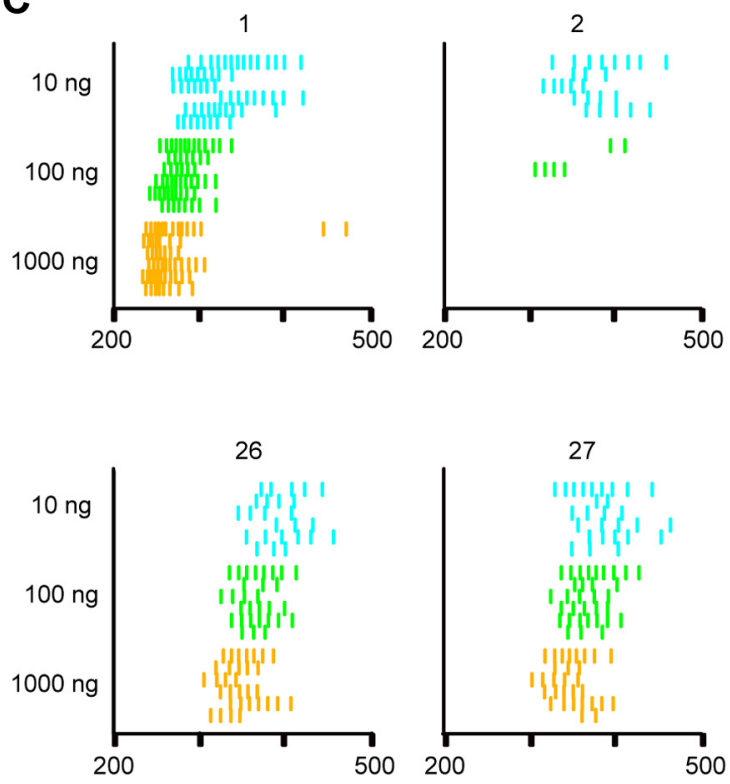

D

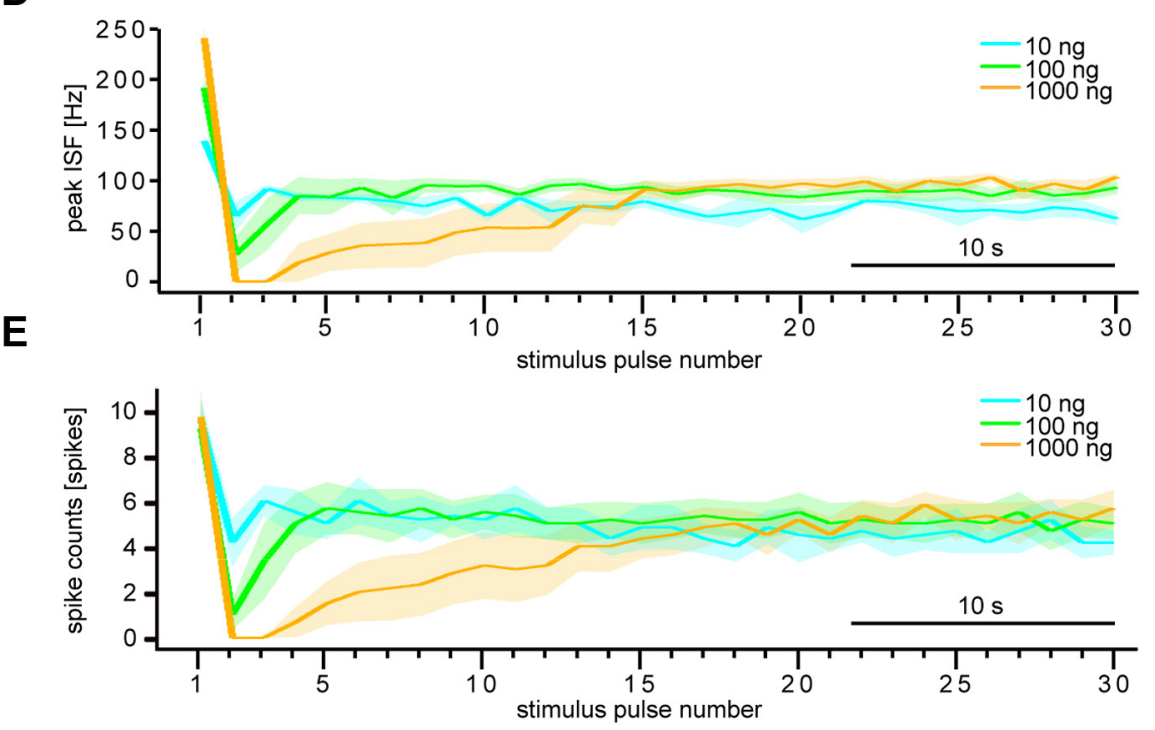

28

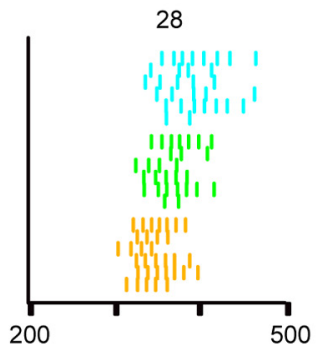

time since stimulus onset [ms]

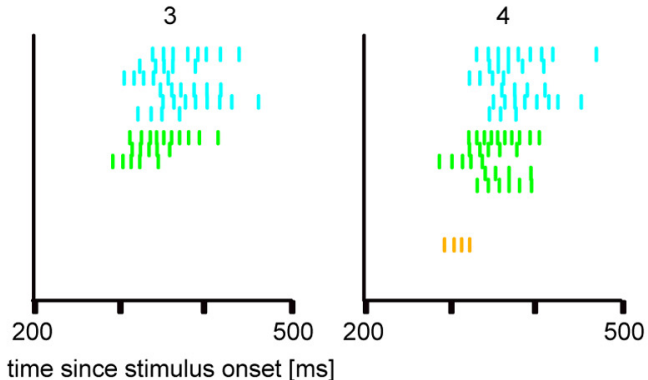

500200

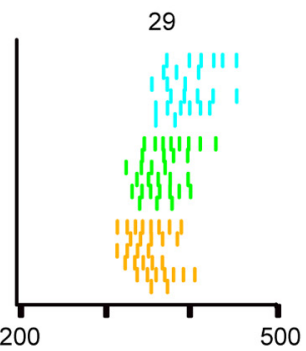

500200

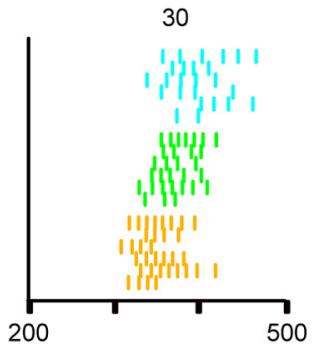

F

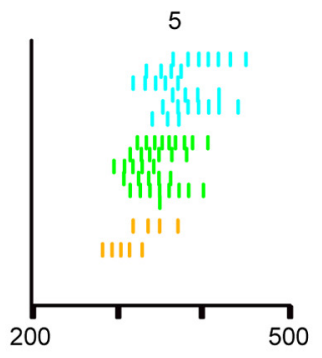

$100 \mu \mathrm{V}$

$00 \mathrm{~ms}$ 
the input of the system) that change approximately threefold in either direction. Just after increasing the concentration, peak ISF and spike counts increased significantly (Fig. $3 A, B ; p<0.05$ between the responses to the 30 th and 31 st stimuli for both peak ISF and spike counts, $n=$ 7). To the subsequent stimuli of the higher concentration, PN responses were suppressed again and finally settled at a constant level comparable with the amplitude before increasing stimulus concentration (Fig. $3 A, B ; p>0.05$ between average settled response levels in response to the lower and higher concentrations for both peak ISF and spike counts, $n=7$ ). When we decreased the stimulus concentration instead, the responses were completely suppressed at first (Fig. 3C,D; $p<0.05$ between the responses to the 30th and 31st stimuli in both peak ISF and spike counts, $n=6$ ) and then gradually recovered (Fig. $3 C, D ; p>0.05$ between average settled response levels in response to the higher and lower concentrations for both peak ISF and spike counts, $n=6$ ). These results support the idea that PNs function as odorant concentration differentiators and encode concentration changes rather than absolute concentrations under intermittent stimulus conditions.

\section{Origin of the transformation of odorant concentration representation}

Where is pheromone response differentiation occurring? To investigate the responses of ORNs to bombykol stimuli, we generated a transgenic moth line expressing GCaMP exclusively in BmOR1expressing neurons, the bombykol-responsive ORNs (henceforth just ORNs) (Fig. 4A,B). The calcium responses in the terminal arborizations of ORN axons to single bombykol pulses increased in a concentration-dependent manner (Fig. $4 C, D)$. When using intermittent stimuli, ORN responses were highest to the first stimulus and reduced for subsequent stimuli of high concentration (Fig. $4 E-G$ ). Although response levels changed upon intermittent stimulation and settled to a stable level over the course of a number of stimuli of the same concentration, there was a clear difference of response amplitudes to stimuli of different concentrations even in the settled state (Fig. $4 F, G$; $F_{(2,5)}=51.95$, Tukey-Kramer test: $p<$ 0.05 among average settled response levels of all concentrations, $n=6$ ). This indicates that the lack of concentration dependence of $\mathrm{PN}$ responses after settling to a stable level under intermittent stimulation is neither the result of presynaptic

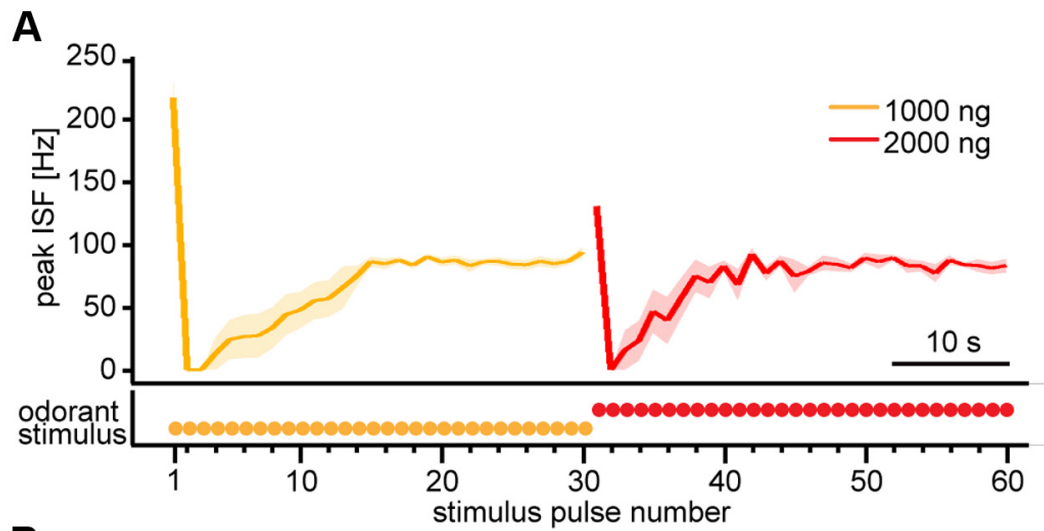

B
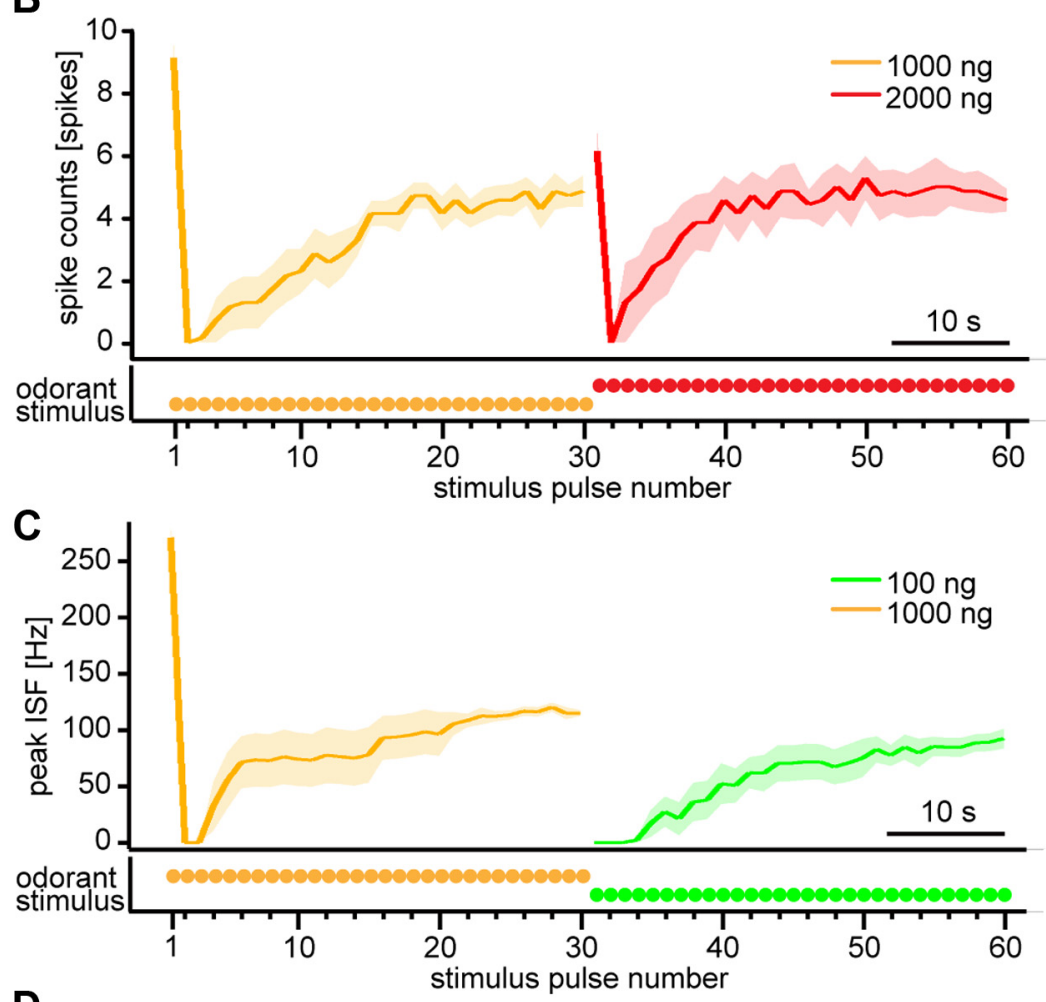

D

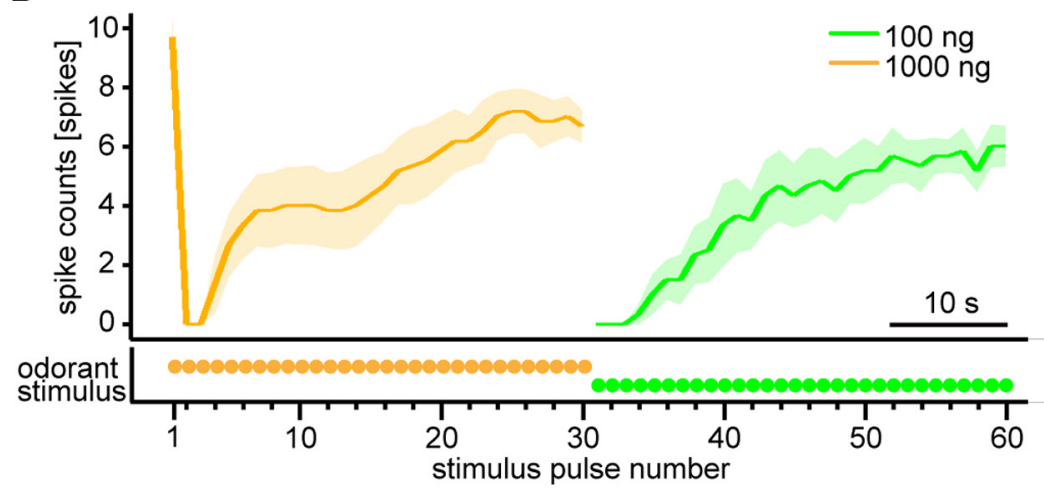

Figure 3. PN responses to intermittent bombykol stimuli upon odorant concentration changes in pulse trains. $\boldsymbol{A}, \boldsymbol{B}$, Dynamics of average peak ISF $(\boldsymbol{A})$ and average spike counts $(\boldsymbol{B})$ of PN responses with odorant concentration increase $(n=$ 7). Odorant concentration was increased from 1000 to $2000 \mathrm{ng}$ after the 30 th stimulus pulse. $C$, D, Dynamics of peak ISF (C) and spike counts $(\boldsymbol{D})$ of PN responses with odorant concentration decrease $(n=6)$. Odorant concentration was decreased from 1000 to $100 \mathrm{ng}$ after the 30th stimulus pulse. The stimulus duration of the pheromone pulses was $200 \mathrm{~ms}$ at $1.2 \mathrm{~s}$ period. Traces below the figures represent stimulus timing and odorant concentration schematically. Shaded areas represent SEM of the responses. 
A
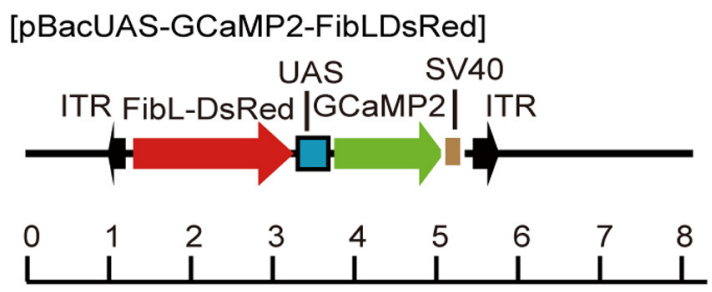

$(\mathrm{kb})$

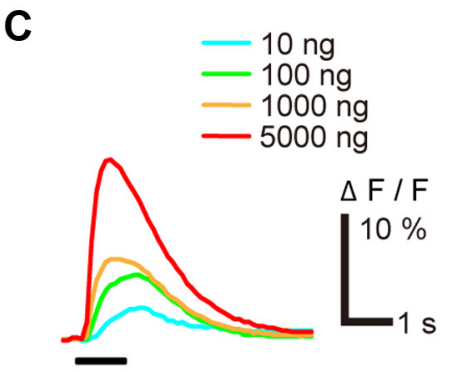

D

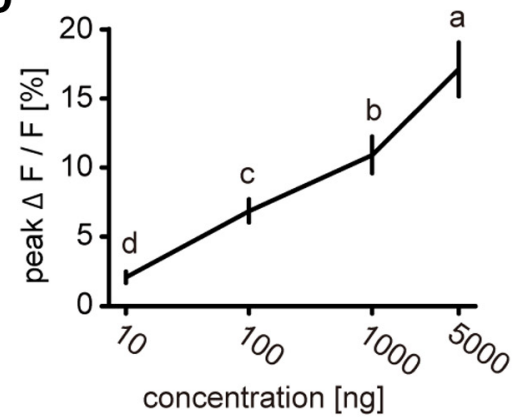

$\mathbf{E}$

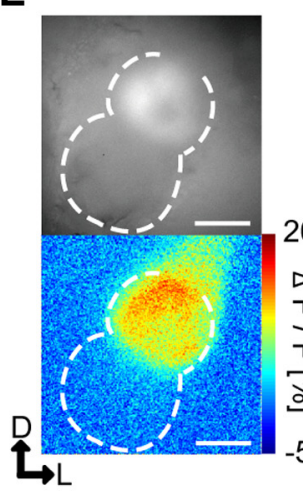

$\mathbf{F}$

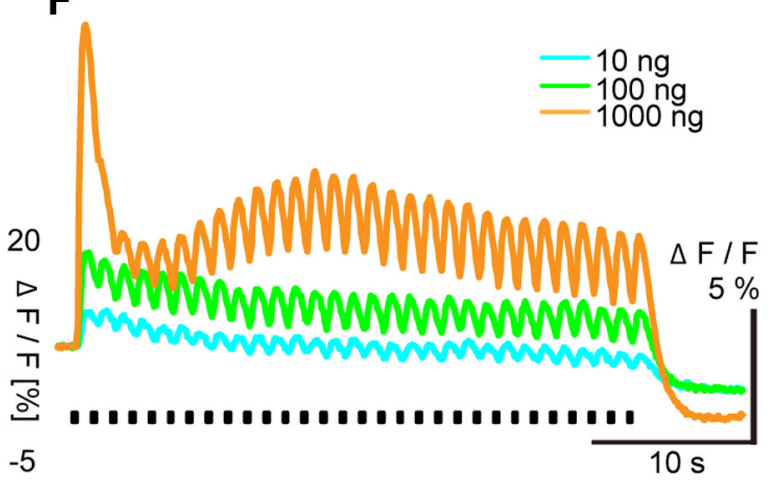

G

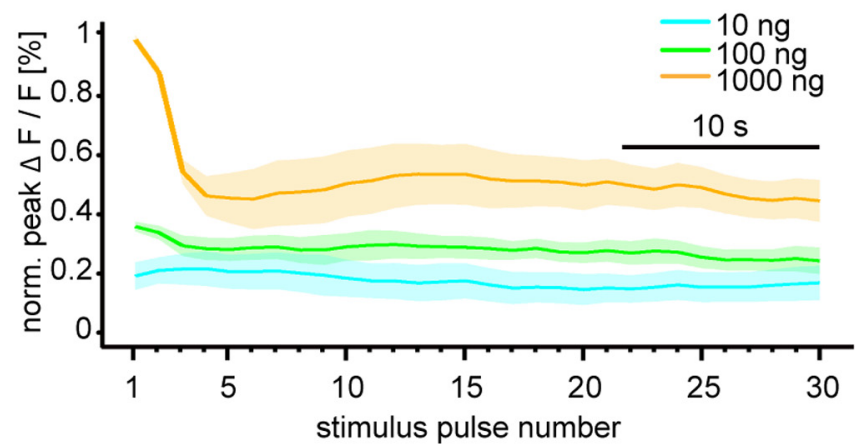

Figure 4. ORN responses to intermittent bombykol stimuli. A, Schematic diagrams of the piggyBac vector, pBacUAS-GCaMP2, used to generate UAS-GCaMP2. FibL-DsRed indicates a screening marker that drives DsRed expression in silk glands. ITR, Inverted terminal repeats of the piggyBac transposon; SV40, SV40 polyadenylation signal. $B$, Representative confocal image of the AL of a BMOR1-GAL4/UAS-GCaMP2 male moth. The axon terminals of bombykol receptor neurons expressing GCaMP (green) were localized in the toroid. Background staining was performed with an anti-synaptotagmin antibody to visualize neuropil structures (magenta). Representative confocal sections are shown. C, Cumulus; T, toroid; D, dorsal; M, medial. Scale bar, $100 \mu \mathrm{m}$. C, Representative GCaMP-expressing neuronal responses to single-pulse bombykol stimulus. Stimulus duration was 1 s. D. Concentrationresponse characteristics of GCaMP-expressing neurons to single-pulse bombykol stimulus (one-way repeated-measures ANOVA, $\left.F_{(3,5)}=48.33, p<0.001\right)$ followed by Tukey-Kramer test $(p<0.05$ for significant differences indicated by different letters associated with the data groups. Data are means. Error bars indicate SEM; $n=6$. $E$, Fluorescence images of the terminal axonal arborization of GCaMP-expressing ORNs (top, averaged for 10 baseline frames) and their responses to $1000 \mathrm{ng}$ bombykol in false colors (bottom). Dashed line indicates the outline of the AL. D, Dorsal; L, lateral. Scale bar, $100 \mu \mathrm{m}$. $\boldsymbol{F}$, Representative time courses of ORN responses to intermittent bombykol stimuli. The stimulus duration of bombykol pulses was $200 \mathrm{~ms}$ at $1.2 \mathrm{~s}$ period. $\boldsymbol{G}$, Dynamics of average normalized peak amplitudes of ORN responses $(n=6)$. Shaded areas represent SEM of the peak amplitudes. mechanisms acting on ORN axon terminals nor a property of ORN responses themselves.

The relative lack of concentration dependence of $\mathrm{PN}$ responses is therefore likely to be generated by other inputs the PNs receive. Previous research in the pheromone-processing circuit of sphinx moth, Manduca sexta, demonstrated that inhibition relayed via $\gamma$-aminobutyric acid type A receptors $\left(\mathrm{GABA}_{\mathrm{A}} \mathrm{Rs}\right)$ in the $\mathrm{AL}$ affects the temporal pattern of $\mathrm{PN}$ responses (Waldrop et al., 1987; Christensen and Hildebrand, 1988; Christensen et al., 1998; Lei et al., 2009). Therefore, we investigated $\mathrm{PN}$ responses during application of PTX, a GABA ${ }_{\mathrm{A}} \mathrm{R}$ antagonist. To the first stimulus, the spike counts were elevated under PTX at medium and high concentrations (Figs. 2C,E and $5 A, C ; p<$ 0.05 PTX against control at both 100 and $1000 \mathrm{ng}, n=6$ ), whereas the peak ISF did not increase (Figs. $2 C, D$ and $5 A, B ; p>$ 0.05 PTX against control, at all concentrations, $n=6$ ). Comparing the raster plots before and during application of PTX shows that inhibition controls the spike counts of responses by shortening the response duration (Figs. $2 C$ and $5 A$; response durations were $84.9 \pm 12.7,58.0 \pm$ 7.0 , and $80.8 \pm 30.6 \mathrm{~ms}$ at 10,100 , and 1000 ng bombykol, respectively, under control conditions vs $104.3 \pm 10.4$, $127.7 \pm 17.4$, and $487.2 \pm 137.4 \mathrm{~ms}$ at 10 , 100 , and $1000 \mathrm{ng}$ bombykol, respectively, under PTX, $p<0.05$ comparing PTX with control conditions at both 100 and 1000 ng bombykol, $n=6$ ). The latency of the last spike after stimulus onset was longer under PTX than under control conditions (Figs. $2 C$ and $5 A ; 369.0 \pm 18.5,311.5 \pm$ 6.7 , and $317.7 \pm 30.8 \mathrm{~ms}$, at 10, 100, and 1000 ng bombykol, respectively, under control conditions vs $407.4 \pm 14.2$, $392.7 \pm 18.4$, and $737.2 \pm 135.2 \mathrm{~ms}$ at 10 , 100 , and $1000 \mathrm{ng}$, respectively, under PTX, $p<0.05$ at all concentrations, $n=$ $6)$. To the subsequent stimuli, response suppression at medium and high concentrations was reduced under PTX, and the peak ISF and the spike counts became concentration-dependent even after settling to a stable level (Fig. $5 A-C$, for peak ISF, $F_{(2,5)}=30.47$, Tukey-Kramer test: $p<0.05$ among average settled response levels for all concentrations; for spike counts, $F_{(2,5)}=33.79$, Tukey-Kramer test: $p<0.05$ among average settled response levels for all concentrations, $n=$ $6)$. The concentration dependence of $\mathrm{PN}$ responses in the settled state during application of PTX was comparable with that of ORN responses (Fig. $5 D, E ; p>0.05$, 
A
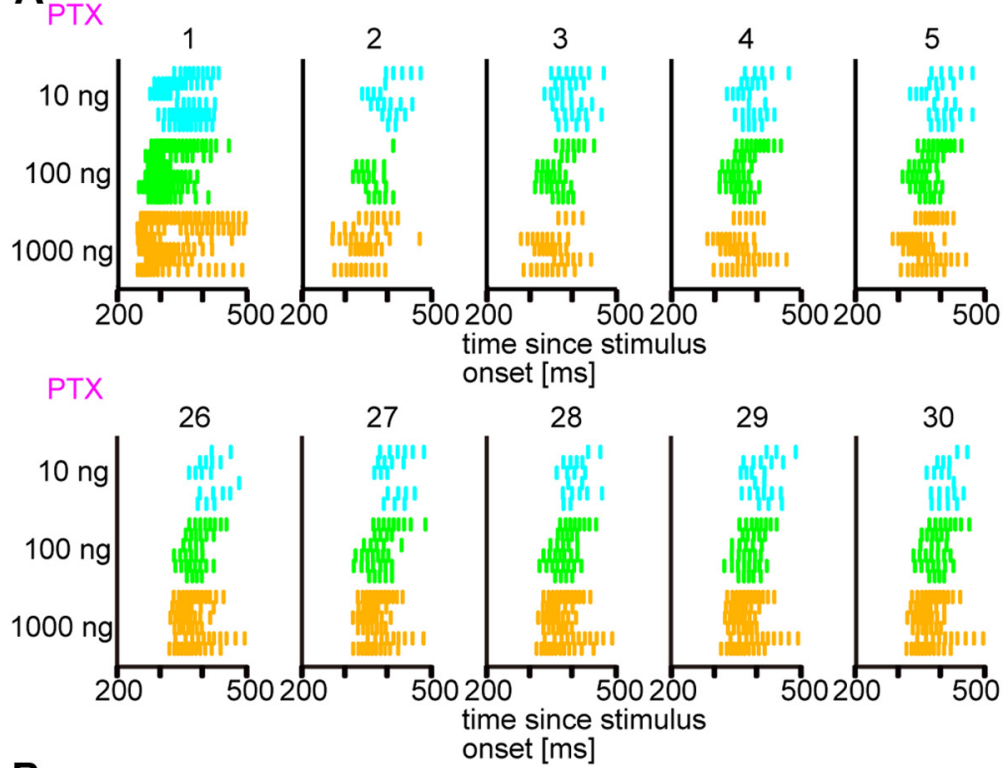

B

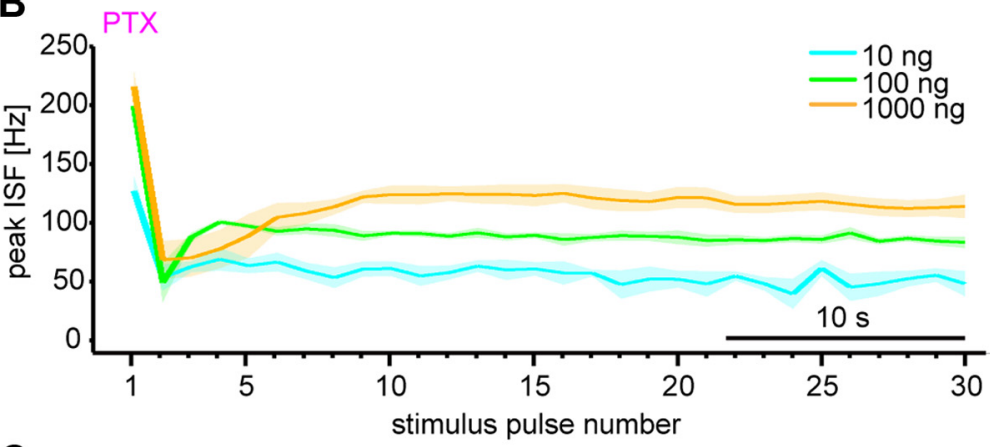

C

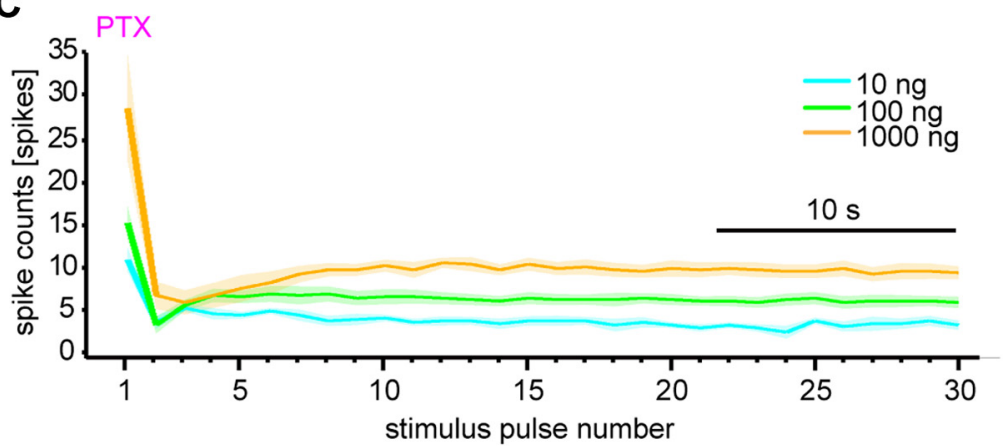

D

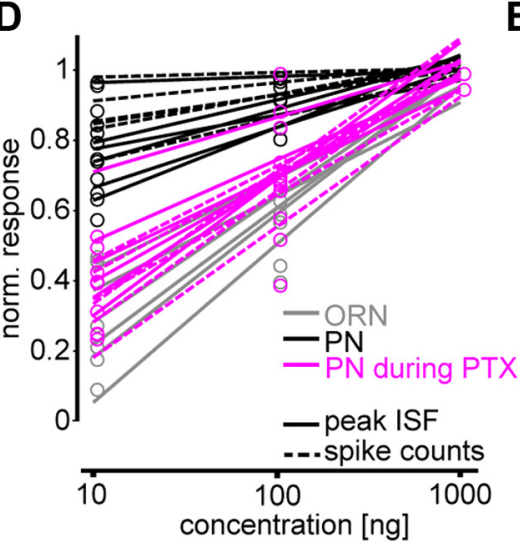

E

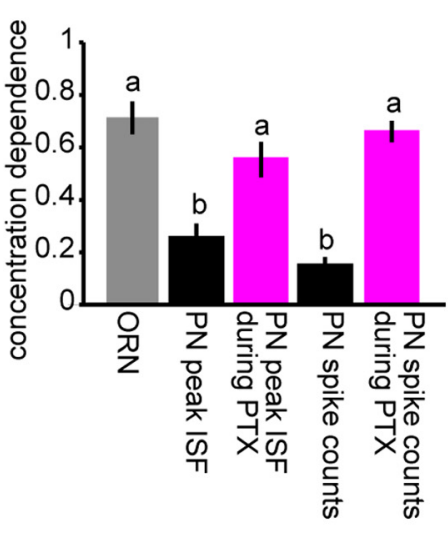

Figure 5. Responses of PNs to intermittent bombykol stimuli under $\mathrm{GABA}_{A}$ receptor blockage with PTX. A, Raster plots of PN responses to the first through fifth stimulus pulses (top) and to the 26th through 30th pulses (bottom) during application of PTX

$n=6)$, although it was significantly lower under control conditions (Fig. $5 D, E$; oneway ANOVA, $F_{(4,5)}=22.05$, TukeyKramer test: $p<0.05, n=6)$. Together with the ORN responses, these results imply that postsynaptic inhibition clamps PN response levels in the settled state.

During application of PTX, the sensitivity of PN responses to concentration changes was partially lost in the paradigm with odorant concentration increase (Fig. $6 A, B)$. The increase of peak ISF upon concentration increase (calculated as the ratio of the responses to 30th and 31st stimulus pulses) was significantly smaller under PTX than under control conditions (Fig. $6 A ; p<0.05, n=8$ ). Although the increase of spike counts upon concentration increase was comparable between pharmacological conditions (Fig. $6 B ; p=$ $0.95, n=8$ ), the average settled level of spike counts at the higher concentration was significantly higher under PTX than in the controls (Fig. $6 B ; p<0.01, n=8$ ) and became comparable with the counts just after increasing concentration (Fig. $6 B ; p=0.84, n=8$ ), although there was a significant difference under control conditions (Fig. $6 B ; p<0.01, n=8$ ). These results imply that an inhibitory mechanism sensitive to PTX is involved in clamping $\mathrm{PN}$ response levels in the settled state and thereby enhancing the responses upon odorant concentration increase in relative terms. Response suppression in PNs after decreasing the odorant concentration was not abolished under PTX (Fig. 6C,D; no spike responses in all PNs just after decreasing concentration in both pharmacological conditions, $n=6$ ), suggesting that a PTX-independent mechanism is responsible for response suppression upon decrease of odorant concentration.

\section{$\leftarrow$}

$(n=6)$. B, C, Dynamics of average peak ISF $(\boldsymbol{B})$ and average spike counts $(\boldsymbol{C})$ in PN responses during application of PTX $(n=6$; shaded areas represent SEM). $\boldsymbol{D}$, Linear fits of normalized ORN responses from imaging data (gray) and normalized PN responses described by peak ISF (solid line) and spike counts (dashed line) before (black) and during application of PTX (magenta). The normalized responses calculated from average settled response levels to last 10 stimulus pulses at each odorant concentration are shown as circles. Values were normalized within each set of odorant concentrations for each parameter and pharmacological condition. $\boldsymbol{E}, 0$ dorant concentration dependencies corresponding to the slopes of fitted curves in $\boldsymbol{D} . p<0.05$, significant differences indicated by different letters associated with the data groups (one-way ANOVA followed by Tukey-Kramer test). $n=6$; data are mean \pm SEM. 
A

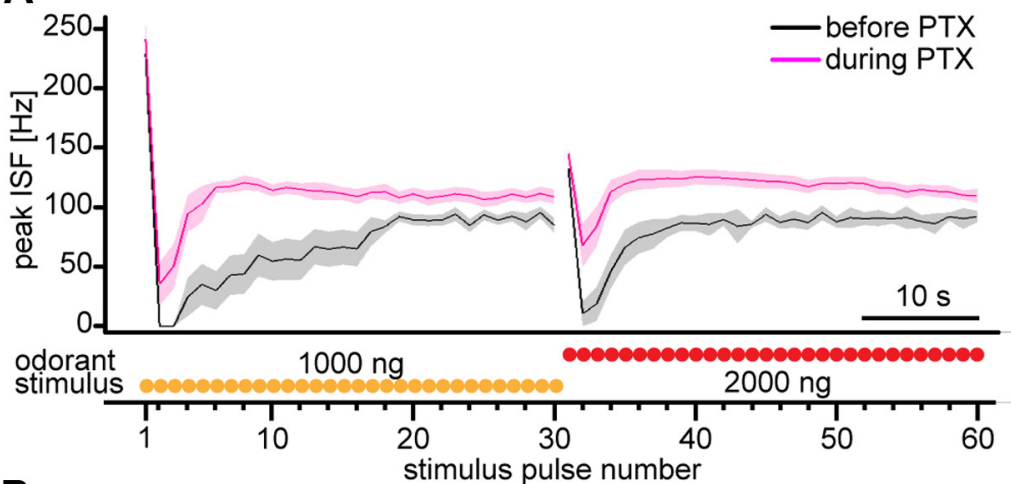

B

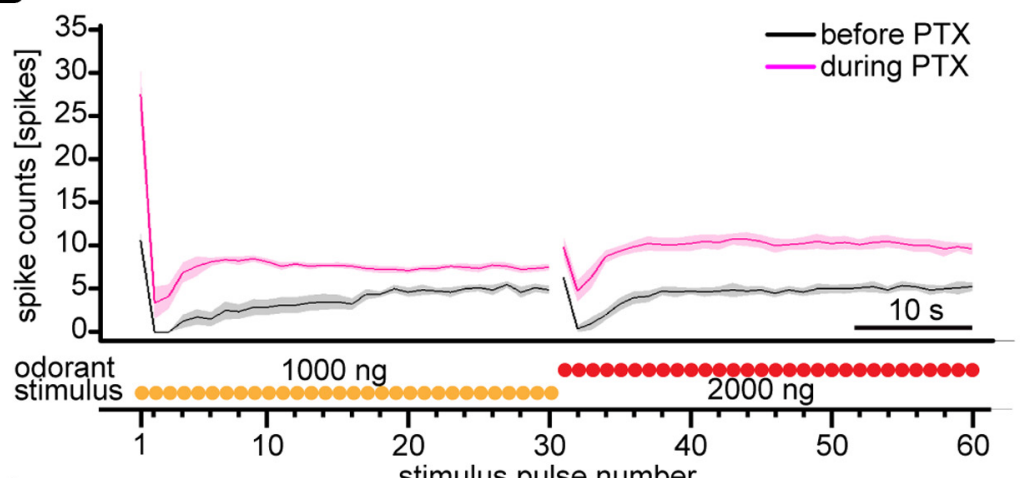

C

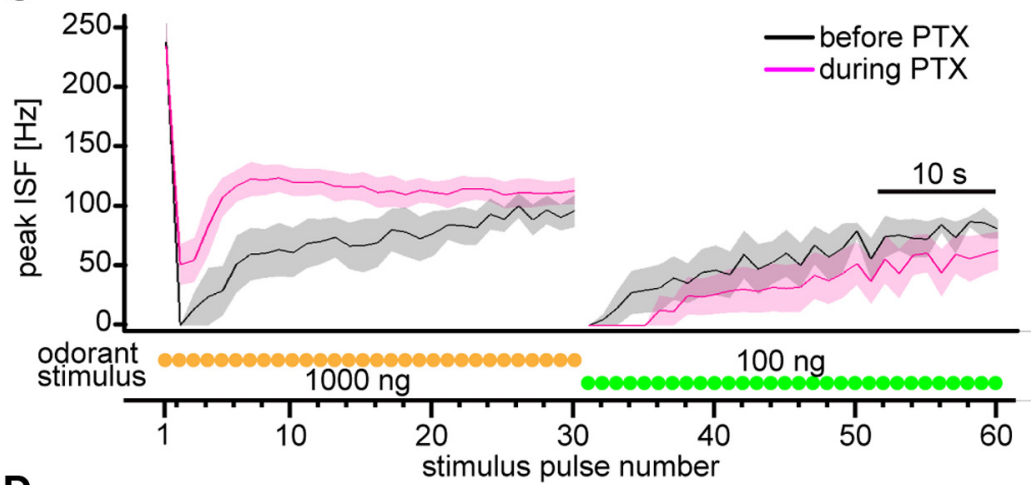

D

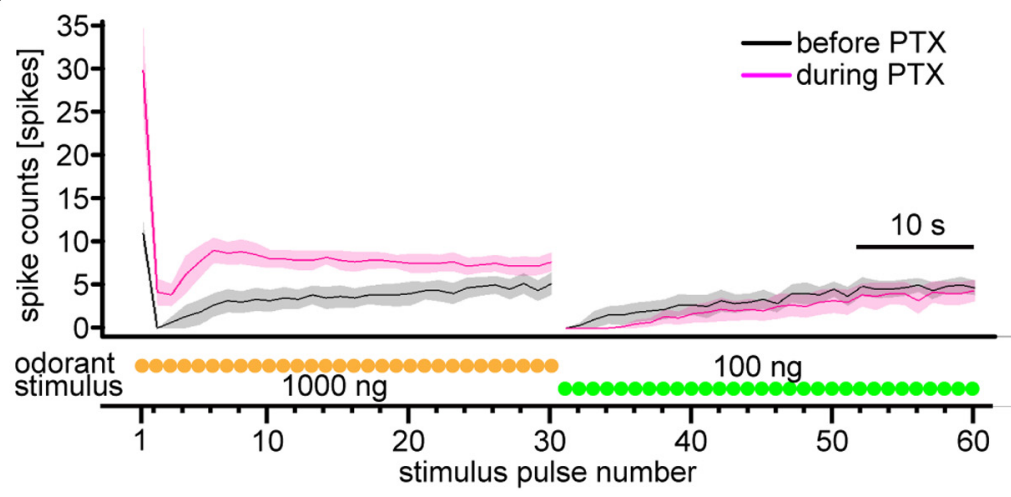

Figure 6. PN responses to intermittent bombykol stimuli upon odorant concentration change in pulse trains and the effect of $G_{A B A} R$ blockade with PTX. $\boldsymbol{A}, \boldsymbol{B}$, Dynamics of average peak ISF $(\boldsymbol{A})$ and average spike counts $(\boldsymbol{B})$ of PN responses with odorant concentration increase before (black) and during (magenta) application of PTX ( $n=8$; shaded areas represent SEM). Odorant concentration was increased from 1000 to $2000 \mathrm{ng}$ after the 30th stimulus pulse. $\boldsymbol{C}, \boldsymbol{D}$, Dynamics of average peak ISF (C) and average spike counts ( $\boldsymbol{D}$ ) of PN responses with odorant concentration decrease before (black) and during (magenta) application of PTX ( $n=6$; shaded areas represent SEM). Odorant concentration was decreased from 1000 to $100 \mathrm{ng}$ after the 30 th stimulus pulse. Traces below the figures represent stimulus timing and odorant concentration schematically.

\section{Simulation of PN responses to naturalistic stimuli}

How does inhibition control the transformation of the odorant concentration representation? In response to the first stimulus of a series, inhibition via $\mathrm{GABA}_{\mathrm{A}} \mathrm{R}$ decreases the spike counts rather than the peak ISF at medium and high concentration by complete suppression of the late phase of spike trains (Fujiwara et al., 2014) (Figs. $2 C$ and $5 A$ ), implying the existence of an inhibitory mechanism with delayed onset, slow offset kinetics, and with activation proportional to $\mathrm{AL}$ input activity originating from the ORNs. This is plausible because strong evoked responses in ORNs and PNs to the first stimulus or a stimulus just after increasing odorant concentration were followed by large response suppressions (Figs. $2 C-E$ and $3 A, B$ ). Strong response suppression could result from superposition of direct stimulus-induced inhibition and residual inhibition elicited by previous stimuli at the stimulus interval used. This idea was tested by constructing a model considering an inhibitory mechanism with delayed onset (100 ms delay with respect to the excitatory input to PNs) and slow offset (1200 ms time constant) kinetics (Fig. $7 A, B$ ). The model successfully replicated the dynamics of $\mathrm{PN}$ responses to intermittent stimuli (Fig. 7C) when inhibition was especially recruited at high odorant concentrations (Fig. 7D). During application of PTX in physiological experiments, complete suppression of $\mathrm{PN}$ responses recovered partially and the responses became concentration dependent in the settled state (Figs. 5 and $7 E$ ). These phenomena were reproducible in the model by removing the inhibitory component (Fig. $7 F$ ). The overall response dynamics of the model resembled our physiological results most closely when inhibition was partially removed (Fig. $7 D, G$ ), which is consistent with an incomplete block of inhibition by PTX, as usually achieved by bath application (Fig. 6). Using this model, we investigated whether inhibition would also improve odorant concentration discrimination for naturalistic odorant stimuli as reflected by the dynamics of EAG responses to females emitting pheromones (Fig. 1). With inhibition, PNs showed highly dynamic changes in their firing rates to concentration changes (Fig. $7 \mathrm{H}$ ) and became more sensitive to both concentration increases and decreases compared with PNs in a model without inhibition (odorant concentration discrimination scores for concentration in- 


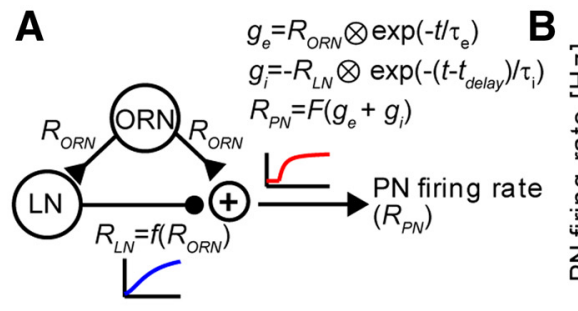

C

experimental data

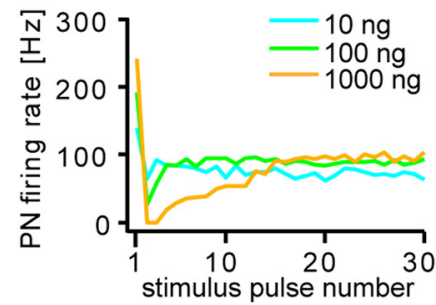

D

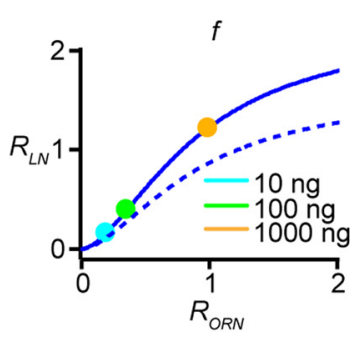

$\mathbf{F}$

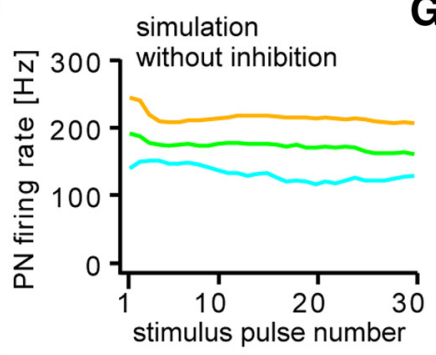

H
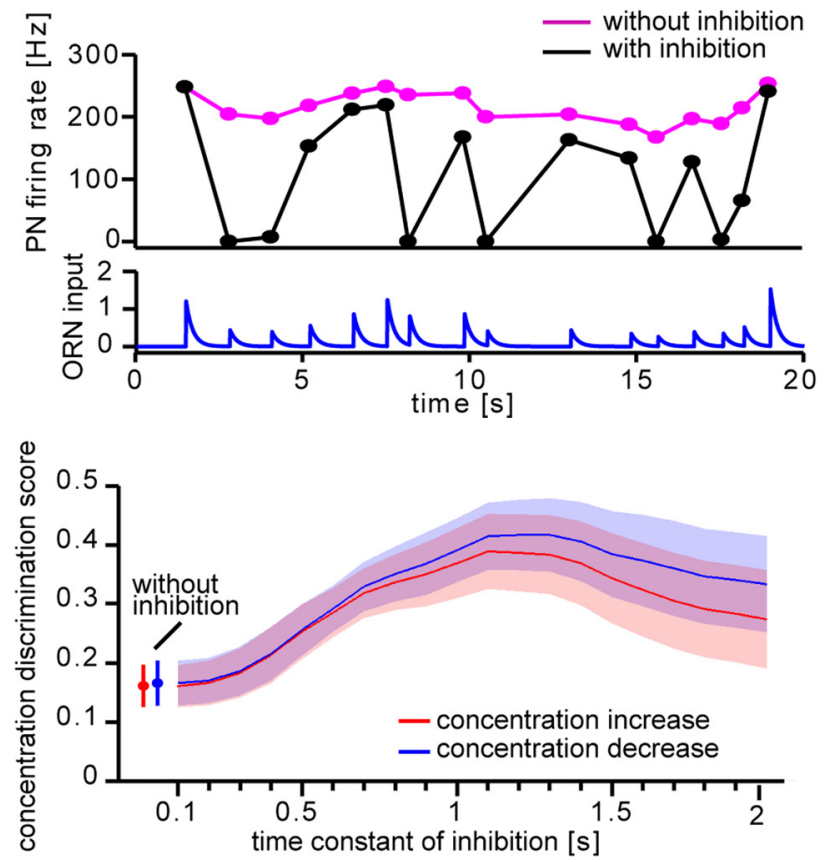

crease were $0.39 \pm 0.07$ with inhibition vs $0.16 \pm 0.04$ without inhibition, $p<0.01$; and scores for concentration decrease were $0.42 \pm 0.06$ with inhibition vs $0.17 \pm$ 0.04 without inhibition, $p<0.01, n=8$ ). Simulation results also imply that this system is matched to the temporal properties of naturally occurring stimuli as concentration discrimination was best when inhibition time constant corresponded to the average interstimulus interval encountered in female-emitted pheromone plumes (1.2 s) (Fig. 7I; optimal time constants of inhibition were $1.1 \mathrm{~s}$ for concentration increase and $1.2 \mathrm{~s}$ for concentration decrease).

\section{Discussion}

In moth pheromone processing systems, dynamic sensory representations have been investigated to characterize the temporal resolution of neuronal responses (Baker et al., 1985; Kaissling, 1986; Christensen and Hildebrand, 1988; Rumbo and Kaissling, 1989; Christensen et al., 1998; Lei and Hansson, 1999; Vickers et al., 2001; Bau et al., 2002; Lei et al., 2009). In this

\section{$\leftarrow$}

Figure 7. Simulation of PN responses. $\boldsymbol{A}$, Schematic diagram of the model. $R_{O R N^{\prime}} R_{L N^{\prime}}$ and $R_{P N^{\prime}}$ Response amplitudes of $0 R N$, LN, and PN, respectively; $g_{e}$ excitatory synaptic conductance of $L N$ and PN activated by ORN input; $g_{i}$, inhibitory synaptic conductance of PN activated by $\mathrm{LN} ; \tau_{\mathrm{e}}$ and $\tau_{\mathrm{i}}$, time constants of excitatory and inhibitory conductances; $t_{\text {delay }}$, $n$ set delay of inhibitory conductance; $t$, time; $F$, transfer function of PN from synaptic conductance to firing rate (shown in $\boldsymbol{B}$ ); $f$, transfer function to determine $L N$ response amplitude (shown in $\boldsymbol{D})$. $\boldsymbol{B}$, Transfer function of PN from synaptic conductance to firing rate. Markers indicate the corresponding odorant concentrations used in the actual experiments at $g_{i}=0 . \max \left(g_{e}\right.$ $\left.+g_{i}\right)$, the local maximum of $g_{e}+g_{i}$ for a stimulus. C, PN responses in the actual experiment (left, same as Fig. 2D) and fitted PN responses in the simulation (right) to intermittent odorant stimuli. D, Transfer function to determine $L N$ response amplitude (solid curve). Markers indicate the corresponding odorant concentrations used in the actual experiments. The transfer function for partial inhibition (dashed curve, corresponding to the result shown in $\boldsymbol{G}$ ) was generated by decreasing the maximum amplitude $\left(R_{L N}\right.$ max $)$ by $30 \%$.E, Electrophysiologically recorded PN responses to intermittent odorant stimuli during application of PTX in the actual experiment to intermittent odorant stimuli (same as Fig. 5B). F, Simulated $\mathrm{PN}$ responses in the simulation to intermittent odorant stimuli without inhibition. $\mathbf{G}$, Simulated PN responses in the simulation to intermittent odorant stimuli with partial inhibition $(70 \%)$. $\boldsymbol{H}$, Representative simulated PN responses to naturalistic odorant stimuli with (black) and without (magenta) inhibition. Traces below the figures show the time course of ORN input to the PN and LN.I, Effect of inhibition time constant on odorant discrimination performance. Shown are average odorant concentration discrimination scores (error bars or shaded areas represent SEM; $n=8$ ) for concentration increase (red) and decrease (blue) for inhibition time constants in the range of $0.1-2 \mathrm{~s}$. 
study, we focused on the representation of odorant concentration rather than on the limits of temporal resolution. Previously, Vickers et al. (2001) showed that EAG amplitude is tightly correlated with both peak ISF and spike counts by simultaneous recording in the tobacco budworm moth, Heliothis virescens, presumably under conditions that correspond to the approach to a female at a larger distance. We estimated the range of pheromone concentrations to which males are exposed in the presence of female-emitted pheromones and used maximum stimulus concentrations in the range of the average concentration received by male antennae. This corresponds to the "high" concentration range we defined in which the concentration-response curve of ORNs is steepest for single stimuli, but in which PNs fail to encode absolute concentration by simply increasing the peak ISF or spike counts upon intermittent stimulation (Figs. 1, 2, and 3). Therefore, the response clamping we observed comes into play in the higher concentration range that corresponds to spatial proximity to a female, and this is also supported by the results of the simulations (Fig. 7D).

Inhibition exclusively comes from LNs, the only GABAergic neurons known to innervate the AL (Iwano and Kanzaki, 2005; Seki and Kanzaki, 2008). Several previous reports have shown that inhibitory neurons (LNs in our system) in primary olfactory centers are increasingly recruited at high odorant concentrations to reduce the activities of principal neurons (PNs in our system) for preserving odorant tuning (Olsen et al., 2010; Tan et al., 2010) and preference at behavioral level (Asahina et al., 2009). Interglomerular inhibition in the Drosophila AL scales with total ORN activity and spatially normalizes $\mathrm{PN}$ responses to odorants (Olsen et al., 2010). Our study shows that a somewhat comparable normalization occurs in the time domain, relying on intraglomerular inhibition. The dynamics of neuronal representations have also been investigated in the mouse olfactory bulb and the locust AL (Brown et al., 2005; Spors et al., 2006; Bathellier et al., 2008; Geffen et al., 2009). These studies focused mainly on odorant discrimination across glomeruli in the primary olfactory areas. We have shown here that a different type of response dynamics exists for encoding odorant concentration in a single glomerulus.

The moth pheromone system is anatomically well separated from the general odorant system at the level of the antennal lobe, comprising a set of neighboring glomeruli, the MGC (Hildebrand, 1996; Kanzaki et al., 2003). Although inhibitory LNs link both systems, we have recently shown that pheromone stimulation induces responses localized to the MGC in these interneurons, implying a role in pheromone concentration discrimination rather than in interaction with the general odorant system (Fujiwara et al., 2014). In the sphinx moth M. sexta, the balance of excitation and inhibition in the AL affects the performance of navigation toward pheromone sources (Lei et al., 2009) and flowers (Riffel et al., 2014) by influencing the ability of AL neurons to track the odorant frequency. Interestingly, intraglomerular inhibition in our study restricts $\mathrm{PN}$ response duration (Figs. 2 and 5), thereby appears also involved in the tracking ability of PNs. Although intraglomerular inhibition has been identified in a general odorant system (Root et al., 2008), its involvement in concentration discrimination remains to be investigated. The mechanism we describe in this study has a function analogous to adaptive processes in other sensory modalities (Kohn, 2007; Wark et al., 2007; Rieke and Rudd, 2009; Barbour, 2011). However, in contrast to these previous studies, the adaptive process in the pheromone system works on intermittent input of a relatively low frequency. Although dynamic changes in
ORN concentration-response characteristics have also been shown here for the first time (Fig. 4), processes in ORNs always preserve concentration-response relationships even when adapted by a pulse train of stimuli. Central processes, whose effect we observe in $\mathrm{PN}$ responses, introduce two additional properties to the responses to dynamic pheromone stimuli: complete response suppression and concentration-independent responses, both of which depend on prior history. Because of the large stimulusinduced response suppression, we propose that a form of shortterm memory of previously encountered stimulus magnitude exists and corresponds to the amplitude of long-lasting inhibition. The concentration-independent response level of PNs in the settled state would then represent the balance of the inhibition and the excitation corresponding to the current stimulus amplitude. Simulations showed that PN response dynamics to intermittent stimuli can be replicated using this simple scheme, and odorant concentration discrimination is improved by longlasting inhibition even for highly dynamic inputs as observed under natural conditions (Fig. $7 H, I$ ). Interestingly, discrimination performance in our model depended on the time constant of the inhibition and displayed an optimum when the time constant was close to the average interstimulus interval of the natural stimuli (Fig. 7I). In visual processing, afterhyperpolarization due to a presynaptic mechanism plays a crucial role in the induction of contrast adaptation (Carandini and Ferster, 1997; Manookin and Demb, 2006). In moth AL PNs, this type of afterhyperpolarization has not been identified but long-lasting hyperpolarizations following pheromone triggered spike trains in moth AL PNs (Christensen and Hildebrand, 1987; Kanzaki et al., 1989; Christensen et al., 1993, 1998; Hansson et al., 1994; Jarriault et al., 2009) are likely to reflect the inhibitory processes that are also involved in dynamically controlling PN response level. We recently reported that PTX-sensitive inhibition directly modulates $\mathrm{PN}$ responses in a concentration-dependent manner (Fujiwara et al., 2014), which is consistent with our observation in this study that $\mathrm{PN}$ responses became concentration-independent in the settled state but ORN responses did not. Here, we directly show that postsynaptic odorant concentration-dependent inhibition is involved in controlling the response dynamics of PNs in intermittent stimulus regimens. Complete PN spike suppression following odorant concentration decrease was not abolished by PTX. This implies that an additional inhibitory mechanism, which could not be blocked under our experimental conditions, could play a role in modulating the representation of odorant concentration information. For intermittent stimulus conditions, absolute concentration information can still be relayed to downstream circuitry through PN response latency (Fig. 2G). However, this would require a mechanism that identifies stimulus timing to extract latency information.

In conclusion, we show that inhibition in the male silkmoth AL dynamically modifies the odorant concentration representation of pheromone-responsive PNs during intermittent stimulation, resulting in an efficient representation of odorant concentration change. This transformation in the olfactory circuit is likely to be important to support efficient adaptive odorant source orientation under dynamically changing natural stimulus conditions.

\section{References}

Asahina K, Louis M, Piccinotti S, Vosshall LB (2009) A circuit supporting concentration-invariant odor perception in Drosophila. J Biol 8:9. CrossRef Medline

Baker TC, Roelofs WL (1981) Initiation and termination of oriental fruit 
moth male response to pheromone concentrations in the field. Environ Entomol 10:211-218.

Baker TC, Willis MA, Haynes KF, Phelan PL (1985) A pulsed cloud of sexpheromone elicits upwind flight in male moths. Physiol Entomol 10:257265. CrossRef

Barbour DL (2011) Intensity-invariant coding in the auditory system. Neurosci Biobehav Rev 35:2064-2072. CrossRef Medline

Bathellier B, Buhl DL, Accolla R, Carleton A (2008) Dynamic ensemble odor coding in the mammalian olfactory bulb: sensory information at different timescales. Neuron 57:586-598. CrossRef Medline

Bau J, Justus KA, Cardé RT (2002) Antennal resolution of pulsed pheromone plumes in three moth species. J Insect Physiol 48:433-442. CrossRef Medline

Boeckh J, Boeckh V (1979) Threshold and odor specificity of pheromonesensitive neurons in the deutocerebrum of Antheraea pernyi and Antheraea polyphemus (Saturnidae). J Comp Physiol 132:235-242. CrossRef

Brand AH, Perrimon N (1993) Targeted gene expression as a means of altering cell fates and generating dominant phenotypes. Development 118: 401-415. Medline

Brown SL, Joseph J, Stopfer M (2005) Encoding a temporally structured stimulus with a temporally structured neural representation. Nat Neurosci 8:1568-1576. CrossRef Medline

Butenandt A, Beckmann R, Stamm D, Hecker E (1959) Über den Sexuallockstoff des Seidenspinners Bombyx mori. Reindarstellung und Konstitution. Z Naturforsch 14b:283-284.

Carandini M, Ferster D (1997) A tonic hyperpolarization underlying contrast adaptation in cat visual cortex. Science 276:949-952. CrossRef Medline

Christensen TA, Hildebrand JG (1987) Male-specific, sex pheromoneselective projection neurons in the antennal lobes of the moth Manduca sexta. J Comp Physiol A Neuroethol Sens Neural Behav Physiol 160:553569. CrossRef Medline

Christensen TA, Hildebrand JG (1988) Frequency coding by central olfactory neurons in the sphinx moth Manduca sexta. Chem Senses 13:123130. CrossRef

Christensen TA, Waldrop BR, Harrow ID, Hildebrand JG (1993) Local interneurons and information-processing in the olfactory glomeruli of the moth Manduca sexta. J Comp Physiol A Neuroethol Sens Neural Behav Physiol 173:385-399. Medline

Christensen TA, Waldrop BR, Hildebrand JG (1998) Multitasking in the olfactory system: context-dependent responses to odors reveal dual GABA-regulated coding mechanisms in single olfactory projection neurons. J Neurosci 18:5999-6008. Medline

Friedrich RW, Korsching SI (1997) Combinatorial and chemotopic odorant coding in the zebrafish olfactory bulb visualized by optical imaging. Neuron 18:737-752. CrossRef Medline

Fujiwara T, Kazawa T, Haupt SS, Kanzaki R (2014) Postsynaptic odorant concentration dependent inhibition controls temporal properties of spike responses of projection neurons in the moth antennal lobe. PLoS One 9:e89132. CrossRef Medline

Geffen MN, Broome BM, Laurent G, Meister M (2009) Neural encoding of rapidly fluctuating odors. Neuron 61:570-586. CrossRef Medline

Gomez-Marin A, Stephens GJ, Louis M (2011) Active sampling and decision making in Drosophila chemotaxis. Nat Commun 2:441. CrossRef Medline

Hallem EA, Carlson JR (2006) Coding of odors by a receptor repertoire. Cell 125:143-160. CrossRef Medline

Hansson BS, Anton S, Christensen TA (1994) Structure and function of antennal lobe neurons in the male turnip moth, Agrotis segetum (Lepidoptera, Noctuidae). J Comp Physiol A Neuroethol Sens Neural Behav Physiol 175:547-562.

Hildebrand JG (1996) Olfactory control of behavior in moths: central processing of odor information and the functional significance of olfactory glomeruli. J Comp Physiol A Neuroethol Sens Neural Behav Physiol 178:5-19.

Iwano M, Kanzaki R (2005) Immunocytochemical identification of neuroactive substances in the antennal lobe of the male silkworm moth Bombyx mori. Zoolog Sci 22:199-211. CrossRef Medline

Jarriault D, Gadenne C, Rospars JP, Anton S (2009) Quantitative analysis of sex-pheromone coding in the antennal lobe of the moth Agrotis ipsilon: a tool to study network plasticity. J Exp Biol 212:1191-1201. CrossRef Medline
Justus KA, Murlis J, Jones C, Cardé RT (2002) Measurement of odor-plume structure in a wind tunnel using a photoionization detector and a tracer gas. Environ Fluid Mech 2:115-142. CrossRef

Kaissling KE (1986) Chemo-electrical transduction in insect olfactory receptors. Annu Rev Neurosci 9:121-145. CrossRef Medline

Kaissling KE, Kasang G (1978) A new pheromone of silkworm moth Bombyx mori. Naturwissenschaften 65:382-384. CrossRef

Kanzaki R, Shibuya T (1983) Olfactory neural pathway and sexual pheromone responses in the deutocerebrum of the male silkworm moth, Bombyx mori (Lepidoptera: Bombycidae). Appl Entomol Zool 18:131-133.

Kanzaki R, Shibuya T (1986) Identification of the deutocerebral neurons responding to the sexual pheromone in the male silkworm moth brain. Zool Sci 3:409-418.

Kanzaki R, Arbas EA, Strausfeld NJ, Hildebrand JG (1989) Physiology and morphology of projection neurons in the antennal lobe of the male moth Manduca sexta. J Comp Physiol A Neuroethol Sens Neural Behav Physiol 165:427-453. CrossRef Medline

Kanzaki R, Soo K, Seki Y, Wada S (2003) Projections to higher olfactory centers from subdivisions of the antennal lobe macroglomerular complex of the male silkmoth. Chem Senses 28:113-130. CrossRef Medline

Khan AG, Sarangi M, Bhalla US (2012) Rats track odour trails accurately using multi-layered strategy with near-optimal sampling. Nat Commun 3:703. CrossRef Medline

Kloppenburg P, Ferns D, Mercer AR (1999) Serotonin enhances central olfactory neuron responses to female sex pheromone in the male sphinx moth Manduca sexta. J Neurosci 19:8172-8181. Medline

Kohn A (2007) Visual adaptation: physiology, mechanisms, and functional benefits. J Neurophysiol 97:3155-3164. CrossRef Medline

Lei H, Hansson BS (1999) Central processing of pulsed pheromone signals by antennal lobe neurons in the male moth Agrotis segetum. J Neurophysiol 81:1113-1122. Medline

Lei H, Riffell JA, Gage SL, Hildebrand JG (2009) Contrast enhancement of stimulus intermittency in a primary olfactory network and its behavioral significance. J Biol 8:21. CrossRef Medline

Manookin MB, Demb JB (2006) Presynaptic mechanism for slow contrast adaptation in mammalian retinal ganglion cells. Neuron 50:453-464. CrossRef Medline

Martelli C, Carlson JR, Emonet T (2013) Intensity invariant dynamics and odor specific latencies in olfactory receptor neuron response. J Neurosci 33:6285-6297. CrossRef Medline

Meeks JP, Arnson HA, Holy TE (2010) Representation and transformation of sensory information in the mouse accessory olfactory system. Nat Neurosci 13:723-730. CrossRef Medline

Olsen SR, Bhandawat V, Wilson RI (2010) Divisive normalization in olfactory population codes. Neuron 66:287-299. CrossRef Medline

Rieke F, Rudd ME (2009) The challenges natural images pose for visual adaptation. Neuron 64:605-616. CrossRef Medline

Riffell JA, Shlizerman E, Sanders E, Abrell L, Medina B, Hinterwirth AJ, Kutz JN (2014) Flower discrimination by pollinators in a dynamic chemical environment. Science 344:1515-1518. CrossRef Medline

Root CM, Masuyama K, Green DS, Enell LE, Nässel DR, Lee CH, Wang JW (2008) A presynaptic gain control mechanism fine tunes olfactory behavior. Neuron 59:311-321. CrossRef Medline

Rumbo ER, Kaissling KE (1989) Temporal resolution of odor pulses by 3 types of pheromone receptor-cells in Antheraea polyphemus. J Comp Physiol A Neuroethol Sens Neural Behav Physiol 165:281-291. CrossRef

Sakurai T, Mitsuno H, Haupt SS, Uchino K, Yokohari F, Nishioka T, Kobayashi I, Sezutsu H, Tamura T, Kanzaki R (2011) A single sex pheromone receptor determines chemical response specificity of sexual behavior in the silkmoth Bombyx mori. PLoS Genet 7:e1002115. CrossRef Medline

Sakurai T, Nakagawa T, Mitsuno H, Mori H, Endo Y, Tanoue S, Yasukochi Y, Touhara K, Nishioka T (2004) Identification and functional characterization of a sex pheromone receptor in the silkmoth Bombyx mori. Proc Natl Acad Sci U S A 101:16653-16658. CrossRef Medline

Seki Y, Kanzaki R (2008) Comprehensive morphological identification and GABA immunocytochemistry of antennal lobe local interneurons in Bombyx mori. J Comp Neurol 506:93-107. CrossRef Medline

Spors H, Wachowiak M, Cohen LB, Friedrich RW (2006) Temporal dynamics and latency patterns of receptor neuron input to the olfactory bulb. J Neurosci 26:1247-1259. CrossRef Medline

Takasaki T, Namiki S, Kanzaki R (2012) Use of bilateral information to 
determine the walking direction during orientation to a pheromone source in the silkmoth Bombyx mori. J Comp Physiol A Neuroethol Sens Neural Behav Physiol 198:295-307. CrossRef Medline

Tallini YN, Ohkura M, Choi BR, Ji G, Imoto K, Doran R, Lee J, Plan P, Wilson J, Xin HB, Sanbe A, Gulick J, Mathai J, Robbins J, Salama G, Nakai J, Kotlikoff MI (2006) Imaging cellular signals in the heart in vivo: cardiac expression of the high-signal $\mathrm{Ca}^{2+}$ indicator GCaMP2. Proc Natl Acad Sci U S A 103:4753-4758. CrossRef Medline

Tamura T, Thibert C, Royer C, Kanda T, Abraham E, Kamba M, Komoto N, Thomas JL, Mauchamp B, Chavancy G, Shirk P, Fraser M, Prudhomme JC, Couble P, Toshiki T, Chantal T, Corinne R, Toshio K, Eappen A, Mari $\mathrm{K}$, et al. (2000) Germline transformation of the silkworm Bombyx mori L. using a piggyBac transposon-derived vector. Nat Biotechnol 18:81-84. CrossRef Medline

Tamura T, Kuwabara N, Uchino K, Kobayashi I, Kanda T (2007) An improved DNA injection method for silkworm eggs drastically increases the efficiency of producing transgenic silkworms. J Insect Biotechnol Sericol 76:155-159.
Tan J, Savigner A, Ma M, Luo M (2010) Odor information processing by the olfactory bulb analyzed in gene-targeted mice. Neuron 65:912-926. CrossRef Medline

Uchino K, Imamura M, Sezutsu H, Kobayashi I, Kojima K, Kanda T, Tamura $\mathrm{T}$ (2006) Evaluating promoter sequences for trapping an enhancer activity in the silkworm Bombyx mori. J Insect Biotechnol Sericol 75:89-97.

Vickers NJ, Christensen TA, Baker TC, Hildebrand JG (2001) Odour-plume dynamics influence the brain's olfactory code. Nature 410:466-470. CrossRef Medline

Waldrop B, Christensen TA, Hildebrand JG (1987) GABA-mediated synaptic inhibition of projection neurons in the antennal lobes of the sphinx moth, Manduca sexta. J Comp Physiol A Neuroethol Sens Neural Behav Physiol 161:23-32. CrossRef Medline

Wang JW, Wong AM, Flores J, Vosshall LB, Axel R (2003) Two-photon calcium imaging reveals an odor-evoked map of activity in the fly brain. Cell 112:271-282. CrossRef Medline

Wark B, Lundstrom BN, Fairhall A (2007) Sensory adaptation. Curr Opin Neurobiol 17:423-429. CrossRef Medline 